\title{
Expression of p53 and CD34 in Surface Epithelial Tumors of Ovary
}

\author{
Dr.A.Arjunan ${ }^{1} \|$ Dr.G.S.Thiriveni Balajji ${ }^{2} \|$ Dr.R.Mani ${ }^{3} \|$ Dr.Sudha. ${ }^{4} \|$ \\ Dr.C.Narmadha ${ }^{5} \|$ Dr.Vinuta Malaichamy ${ }^{6} \|$ \\ ${ }^{I}$ ( Professor of Pathology, Pathology Department, Coimbatore Medical College ${ }^{\dagger}$, Tamilnadu, India) \\ ${ }^{2}$ ( Associate Professor, Pathology Department, Coimbatore Medical College ${ }^{\dagger}$, Tamilnadu, India) \\ ${ }^{3}$ ( Professor of Pharmacology, Pharmacology Department, Coimbatore Medical College ${ }^{\dagger}$, Tamilnadu, India) \\ ${ }^{4}$ (Senior Assistant Professor, Pathology Department, Coimbatore Medical College ${ }^{\dagger}$, Tamilnadu, India) \\ ${ }^{5}$ (Assistant Professor, Pathology Department, Coimbatore Medical College ${ }^{\dagger}$, Tamilnadu, India) \\ ${ }^{6}$ (Senior Assistant Professor, Pathology Department, Coimbatore Medical College ${ }^{\dagger}$, Tamilnadu, India) \\ † The Tamilnadu Dr.MGR Medical University, Tamilnadu, India and \\ Tamilnadu State Research Committee, King Institute of Preventive Medicine and Research, Guindy, Chennai-
} 32.

\begin{abstract}
$\overline{\text { Abstract: Ovarian cancer accounts for } 3 \% \text { of all cancers in females }{ }^{[1]} \text {. Owing to diagnostic difficulty of }}$ ovarian cancers at early stage, most of them are diagnosed when they are at metastatic stage, thus they account for increased number of deaths occurring in women due to genital tract tumors. Ovarian Carcinomas are currently divided into Type I ( low grade) and Type II ( high grade) tumours. ${ }^{[1]}$ p53 and CD34 have been reported to play an important role in the progression of ovarian cancer. However, only few studies focus on their expression in benign surface epithelial tumors compared to malignant surface epithelial tumors and further low grade tumors compared to high grade tumors in the malignant category. Tumour angiogenesis, evaluated by CD34 expression as intratumoral microvessel density and CD34 intensity index could be an important predictor of the aggressive nature of high grade ovarian carcinoma. Overexpression of p53 in ovarian tumors of both low-grade and high-grade ovarian tumors has been found to be associated with poor prognosis in most but not all studies. ${ }^{[2]}$ In present study, Mean microvessel density, CD34 intensity index and p53 proportion scoring of High grade tumors was statistically significantly higher than Low grade tumors ( $p$ value <0.05). Infact, immunohistochemical staining for p53 has been utilized as a surrogate marker for mutational analysis in the diagnostic workup of carcinomas of multiple sites including ovarian cancers. ${ }^{[3]}$ Implication of 533 and CD34 in predicting the metastatic potential of ovarian surface epithelial carcinomas has to be further analysed with larger sample size.
\end{abstract}

Keywords: CD 34, high grade, low grade, ovarian cancer, p53

\section{Introduction}

Ovarian Cancer represents the sixth most commonly diagnosed cancer among women in the world. ${ }^{[4]}$ Incidence of Ovarian cancer in India was estimated to be 4.9 per $1,00,000$ population. ${ }^{[5]}$ Within India, Ovarian cancer incidence rates vary considerably, with a strong urban/rural gradient. ${ }^{[6]}$ Neoangiogenesis is the formation of new blood vessels from the pre-existing vascular channels. Generally in tumors including ovarian tumors, angiogenesis is essential for survival of tumor cells and predisposes for metastasis. It can be estimated by analyzing the microvessel density and CD34 intensity index. CD 34 is a vascular endothelial marker that highlights vascular channels. p53 is a marker that indirectly reflects the cell cycle changes in the tissue. Both CD34 and p53 can be demonstrated using immunohistochemistry on formalin fixed and paraffin embedded tissues after antigen retrieval. The present study analyses the expression of CD34 and p53 in benign and malignant ( low grade and high grade) surface epithelial ovarian tumors. This would pave way for further analysis of the prognostic significance of CD34 and p53 in ovarian tumors.

\section{Aim And Objectives}

\subsection{Aim:}

The aim of the present study is to assess expression of CD34 and p53 in benign and malignant and further in low grade(Type I) and high grade( Type II) malignantsurface epithelial tumors of ovary.

\subsection{Objectives:}

a. To estimate microvessel density and p53 expression in 32 cases of benign and 18 cases of malignant surface epithelial tumors of ovary. 
b. To compare the microvessel density and CD34 intensity index among benign and malignant surface epithelial tumors of ovary.

c. To compare 533 proportion score among benign and malignant surface epithelial tumors of ovary.

d. To analyse microvessel density, CD34 intensity index and p53 proportion score in 9 cases low grade and 9 cases of high grade malignant surface epithelial tumors of ovary

3.1 Study design: Analytical study

\section{Materials And Methods}

3.2Study population: 50 cases of surface epithelial tumors of ovary.

3.3 Study period: One year.

3.4 Inclusion criteria:

1. Age between 20 to 60 years of age.

2. All histological types of surface epithelial tumors of ovary received during the period of one year from June,2015 to May 2016 in the Department of Pathology, Coimbatore Medical College Hospital, Coimbatore.

3.5 Exclusion criteria:

1. Age $<20$ years and $>60$ years.

2. Histological types of ovarian neoplasms other than surface epithelial tumors of ovary.

3. Patients already receiving chemotherapy and radiotherapy for ovarian tumors.

4. Recurrent ovarian tumors.

5. Metastatic deposits in ovary.

3.6 Specimen selection and histopathology processing: Surgically removed ovarian tumors were fixed in 10 $\%$ neutral buffered formalin solution. Most representative part of the tumor was selected and tumor bits of size $10 \times 10 \times 3 \mathrm{~mm}$ were isolated. Bits from areas of necrosis and extensive hemorrhage were avoided. The isolated tumor bits were processed for paraffin embedding and stained with routine haematoxylin and eosin stain. The slides were subjected to histopathological evaluation and slides were selected for Immunohistochemical staining.

3.7 Positive control: Tonsil tissues were used as positive control for p53 and internal control of vessels were used for CD34.

3.8 Immunohistochemistry: The slides selected for immunohistochemistry were incubated overnight at 37 degree Centigrade. Subsequently, Antigen retrieval was performed using microwave technique for 20 minutes in Citrate buffer at $\mathrm{pH} 6$.

Later, primary antibody i.e. Mouse monoclonal anti-CD34 antibody and mouse monoclonal anti - p53 antibody were applied on the slides and were incubated at room temperature for one hour. Subsequently, secondary antibody was applied and DAB chromogen was added to the slides.

\subsection{Microscopic Examination:}

Under light microscope, Haematoxylin and Eosin slides were analysed, tumors were classified and graded.Subsequently immunohistochemically stained slides for p53 and CD34 were evaluated for scoring.

At low power (100X) magnification the slides were analyzed to locate "hot spots" i.e. areas of maximal microvessel density (MVD). Brown staining of endothelial cells, clearly separated from adjacent microvessels, connective tissue and tumor cells was considered as a single countable microvessel. At high power (400x ) magnification, in three hot spots, in each case, the microvessel density was assessed. The average microvessel density of three fields at 400x magnification was calculated. Large vessels with thick muscular wall were not included in the count.

Intensity of CD34 expression in endothelial cells in each tumor vessel was also assessed. It was graded into high, moderate, low and absent in comparison to positive control. CD34 intensity index was defined as ratio of tumor vessels with high or moderate intensity of CD34 to number of all countable tumor vessels at 400x magnification. This ratio was calculated in three hot spot areas and average CD34 intensity index was assessed. ${ }^{[7]}$

Proportion of cells expressing strong nuclear positivity for p53 were counted in three foci. Average score of the three foci was noted for each case. Mean value and standard deviation of the score for each histological type was assessed. This was performed particularly in both low-grade and high grade malignant surface epithelial tumors. 
Intensity of staining for p53 was observed as $100 \%$ ( strong positive ) in all cases. Adjacent non-neoplastic cells that stain positive for $\mathrm{p} 53$ were taken as positive control, where it was applicable. Tonsil tissue was subjected to p53 immunostaining, as part of quality control for p53. Proportion of cells staining for p53 were graded as follows :

Grade $1: 0$ to $10 \%$ staining

Grade $2: 11$ to $40 \%$ staining

Grade $3: 41$ to $60 \%$ staining

Grade $4: 61$ to $100 \%$ staining

\subsection{Statistical analysis:}

The results were tabulated and analysed for statistical significance using unpaired t-test. A 'p' value of $<0.05$ was considered to be statistically significance.

\section{Observation And Results}

A total of 50 patients with surface epithelial tumors of ovary received in the Department of Pathology during one year period were analysed in the present study.

Table 1: Age distribution of surface epithelial tumors of ovary

\begin{tabular}{|l|l|}
\hline AGE & NUMBER OF PATIENTS \\
\hline$<20$ years & 0 \\
\hline $21-30$ years & 1 \\
\hline $31-40$ years & 12 \\
\hline $41-50$ years & 14 \\
\hline $51-60$ years & 15 \\
\hline$>60$ years & 8 \\
\hline Total & 50 patients \\
\hline
\end{tabular}

As seen in the above table, patients aged between 51 to 60 years had the maximum incidence (15 patients) of surface epithelial tumors of ovary followed by age 41 to 50 years (14 patients).

Patients with surface epithelial tumors of ovary were classified into benign and malignant group as per WHO classification. ${ }^{[8]}$ Since grading of ovarian carcinoma is not yet standardized ${ }^{[8]}$ we followed the grading system proposed by Cho K R which is as follows : Type I (low grade) tumors include - low grade serous carcinoma, low grade endometrioid carcinoma and mucinous carcinoma, while Type II (high grade) tumors include High-grade serous carcinoma, High grade endometrioid carcinoma, Undifferentiated carcinoma and Carcinosarcoma ${ }^{[9]}$ Criteria for classification of clear cell carcinoma into type I versus type II subsets are uncertain. However, based on degree of nuclear atypia, almost all clear cell carcinoma are considered as Malignant High Grade tumors. ${ }^{[10,11]}$

TABLE 2: Histomorphological distribution of surface epithelial tumors of ovary

\begin{tabular}{|c|c|c|}
\hline HISTOMORPHOLOGICAL TYPE & DIFFERENTIATION OF TUMOR & NUMBER OF PATIENTS \\
\hline Serous cystadenoma & Benign & 18 \\
\hline Serous cystadenofibroma & Benign & 4 \\
\hline Serous cystadenocarcinoma & Malignant, Low grade & 7 \\
\hline Serous cystadenocarcinoma & Malignant, High grade & 10 \\
\hline Mucinous cystadenoma & Benign & 4 \\
\hline Mucinous cystadenocarcinoma & Malignant, Low grade & 1 \\
\hline Endometrioid carcinoma & Malignant, Low grade & 1 \\
\hline Brenner tumor & Benign & 1 \\
\hline Clear cell carcinoma & Malignant, High grade & 1 \\
\hline Undifferentiated & Malignant, High grade & 50 patients \\
\hline
\end{tabular}

Out of 50 patients, 32 patients had benign surface epithelial ovarian tumor while 18 patients had malignant ovarian tumor. In present study, out of the 18 malignant surface epithelial ovarian tumor patients, 9 patients were of low grade and 9 patients were of high grade. 

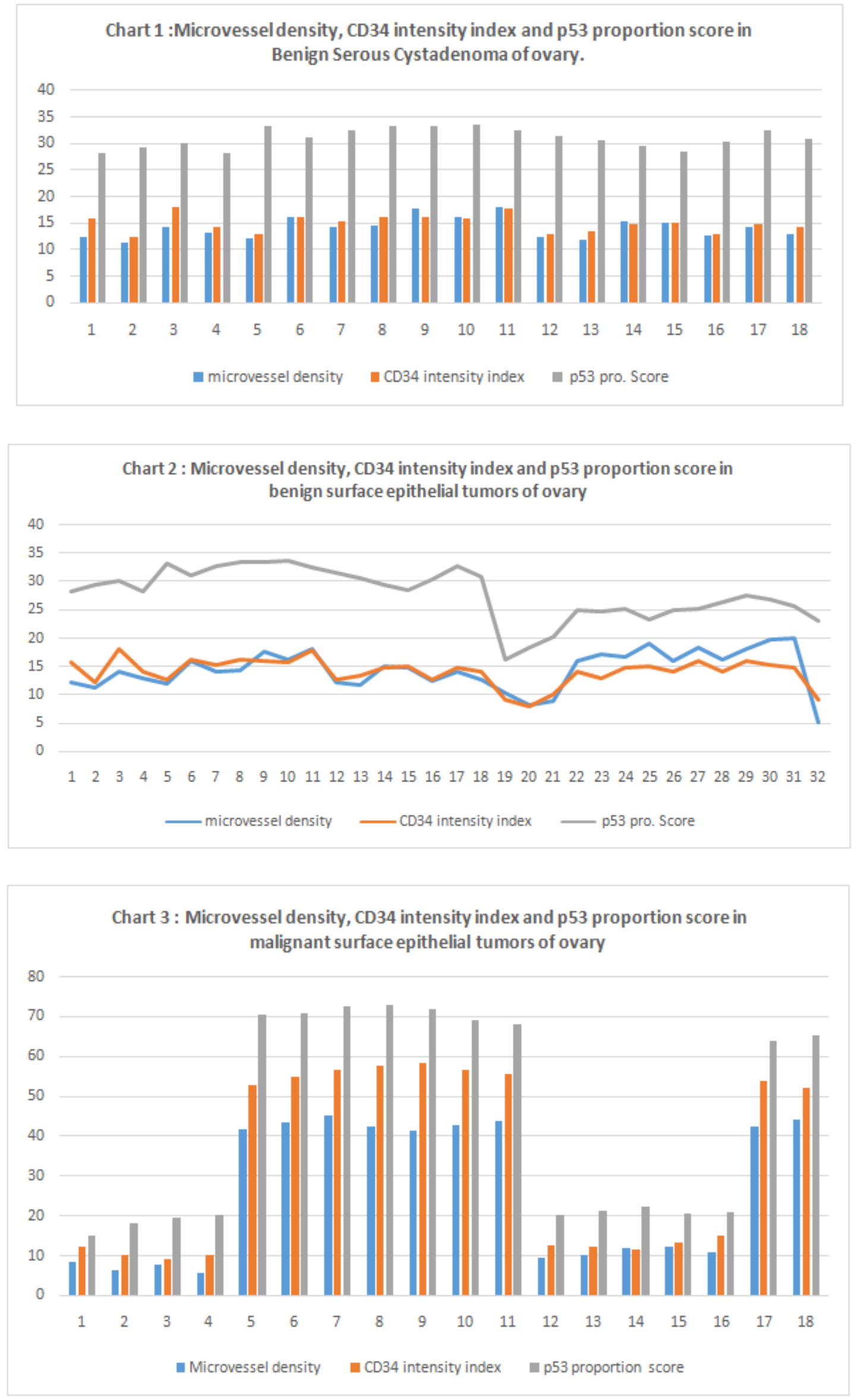
TABLE 3 : Comparision of expression of CD34 and p53 in benign and malignant surface epithelial tumors of ovary

\begin{tabular}{|c|c|c|c|}
\hline PARAMETER & $\begin{array}{c}\text { BENIGN TUMORS } \\
(\mathbf{N = 3 2})\end{array}$ & $\begin{array}{c}\text { MALIGNANT TUMORS } \\
(\mathbf{N = 1 8})\end{array}$ & P VALUE \\
\hline Mean Microvessel Density & 14.88 & 26.09 & 0.002 \\
\hline Mean CD34 intensity index & 14.14 & 33.6 & 0.0001 \\
\hline Mean p53 proportion score & 27.83 & 44.86 & 0.0001 \\
\hline
\end{tabular}

Using unpaired $\mathrm{t}$ test, statistical significance was sought between benign and malignant surface epithelial tumors of ovary. Mean Microvessel Density, Mean CD34 intensity index and Mean p53 proportion score were compared. All three parameters showed $\mathrm{p}$ value less than 0.05 , which indicates statistically significant correlation. Thus, it is observed that malignant surface epithelial ovarian tumors have higher expression of CD34 and p53 compared to benign tumors.

TABLE 4: Comparision of expression of CD34 and p53 in low grade and high grade malignant surface epithelial tumors of ovary

\begin{tabular}{|l|c|c|c|}
\hline PARAMETER & $\begin{array}{c}\text { MALIGNANT LOW } \\
\text { GRADE TUMORS }(\mathbf{N = 9})\end{array}$ & $\begin{array}{c}\text { MALIGNANT HIGH } \\
\text { GRADE TUMORS ( N=9) }\end{array}$ & P VALUE \\
\hline Mean Microvessel Density & 9.1 & 43.07 & 0.0001 \\
\hline Mean CD34 intensity index & 11.72 & 19.2 & 0.0001 \\
\hline Mean p53 proportion score & 19.72 & 69.58 & 0.0001 \\
\hline
\end{tabular}

Using unpaired t test, statistical significance was sought between low grade and high grade malignant surface epithelial tumors of ovary. Mean Microvessel Density, Mean CD34 intensity index and Mean p53 proportion score were compared. All three parameters showed $\mathrm{p}$ value less than 0.05 , which indicates statistically significant correlation. Thus, it is observed that high grade tumors have higher expression of CD34 and p53 compared to low grade tumors.

\section{Discussion}

5.a In the yesteryears, ovarian cancers has been graded as well, moderate and poorly differentiated prompting that similar histogenesis is applied for all the three grades. However, recent studies refer to two tier grading system i.e. low- grade and high-grade with different histogenesis pattern. The various grading systems include the following. ${ }^{[12]}$

Table 5 : Various Grading Systems of Ovarian tumors

\begin{tabular}{|l|l|l|}
\hline 1. & FIGO system & Based on the percentage of solid architecture \\
\hline 2. & WHO system & Based on both architecture and cytology \\
\hline 3. & GOG(Gynaecologic Oncology Group ) & Based on histologic type \\
\hline 4. & Others (1) & Based on a combination of mitotic index and volume percentage of epithelium \\
\hline 5. & Others (2) & $\begin{array}{l}\text { Based on presence / amount of hyperchromatic giant nuclei and solid/ cribriform } \\
\text { architecture. }\end{array}$ \\
\hline 6. & Others ( 3) & $\begin{array}{l}\text { Grading index based on a mean of the individual scores for architectural pattern, } \\
\text { nuclear pleomorphism, nucleoli, nuclear }- \text { to -cytoplasmic ratio, mitotic index, } \\
\text { pattern of invasion, capsule penetration and vascular invasion }\end{array}$ \\
\hline 7. & $\begin{array}{l}\text { Silverberg grade (or) Universal } \\
\text { grading system }\end{array}$ & $\begin{array}{l}\text { Points are assigned for 3 components : architecture ( glandular, papillary or solid), } \\
\text { nuclear atypia and mitotic index. Points for each of the three components are added to } \\
\text { get the total score that determines the score. }\end{array}$ \\
\hline
\end{tabular}

In the recent years, a two tier grading system has been proposed for malignant surface epithelial tumors of the ovary, such as, Low-grade(Type I) and High grade( Type II) surface epithelial tumors of ovary. Both the grades have distinct pathogenesis, clinicopathological, immunohistochemical and molecular profiles. Application of immunostains including p53, Ki67, p16 and CD34 are found to be useful in distinguishing such cancers as low-grade and high-grade carcinomas.

5.b Angiogenesis is the formation of new blood vessels that plays a pivotal role in progression and dissemination of ovarian tumors. The development of new capillaries is finely regulated by the balance between activators and inhibitors of angiogenesis. When a tumor becomes high grade, it is associated with accelerated neoangiogenesis. Thus, there exists an imbalance between increased angiogenic stimulatory factors and decreased angiogenic inhibitory factors.

As many as 20 different activators and inhibitors of angiogenesis have been described. ${ }^{[13]}$ Few of them include acid and basic Fibroblast Growth Factors ( FGF) have a direct mitogenic effect. Some others like Tumor Necrosis Factor (TNF) and Transforming Growth Factor - alpha (TNF-alpha) activate macrophages, thus stimulating mitogenic production. Of all the factors, Vascular Endothelial Growth Factor (VEGF) is described as the most specific activator for development of new capillaries. Angiogenic stimulatory factors are synthesized 
and secreted by the new vessels, surrounding tumor cells, fibroblasts, mastocytes, granulocytes, macrophages and lymphocytes. ${ }^{[14]}$

5.c CD34, an angiogenic factor is a cell surface protein which is a marker of haematopoetic progenitor cells. CD34 expression in bone marrow cells is low and further decreases during haematopoetic differentiation. CD34 is also expressed in endothelial cells. Upregulation of CD34 is associated with increased neovascularization during tumor development. The technique of immunohistochemistry can be applied to tissue sections of tumors to estimate the expression of CD34.

The quantity of vessels observed within the tumor could indicate the level of angiogenic activity in the tumor. Accelerated neovascularization is associated with increased expression of CD34 during tumor development. Many studies have been undertaken to analyse the prognostic significance of microvessel density and CD34 intensity index of new vessels.

According to Folkman J., growth of tumors more than $2 \mathrm{~mm}$ in diameter, requires activation of angiogenic factors and thus accelerates neovascularization to maintain blood supply. Neovascularisation, to begin with, starts as small capillary channels, branching from vessels situated in the normal tissue surrounding the tumor. Later malignant tissue produces angiogenic stimulatory factors including cytokines, leading to proliferation and formation of new capillaries by endothelial cells. ${ }^{[15]}$

However, in a study by Heimburg et al, they did not notice any significant correlation between CD34 expression and microvessel density, histological type or the grade of investigated cancer. ${ }^{[16]}$

In a research study of 80 patients with ovarian carcinoma by Rossachacka- Rostalka B et al, it was found that independent risk factors of death in patients with ovarian cancer included age over 50 years, low degree of patient performance according to WHO scale, high FIGO stage and high expression of CD34 microvessel density. ${ }^{[17]}$

In present study, Mean microvessel density and CD34 intensity index of malignant tumors was statistically significantly higher than benign tumors ( $p$ value <0.05). A similar observation was seen in High grade tumors compared to Low grade tumors in malignant ovarian surface epithelial carcinomas.In studies by Muthuramalingam SR, et al, ${ }^{[18]}$ Matulonis UA, ${ }^{[19]}$ Hata K, et al, ${ }^{[20]}$ the importance of antiangiogenic therapy in ovarian cancer was observed.

5.d The p53 protein is also known as the " the Guardian of Genome " and it is an activator of transcription. In normal state, it binds to DNA sequences in the control region, thus affecting their expression. Binding of p53 to DNA sequences leads to expression of specific genes necessary for inhibition of cell growth i.e. apoptosis. ${ }^{[21]}$ Alterations of p53 activity, occurring due to point mutations or deletions or due to protein stabilization are commonly seen in cancers. ${ }^{[22]}$ These mutations lead to the loss of wild -type p53 function and thus lead to uncontrolled growth of affected cells.

Infact, accumulation of p53(mutant) protein has been related to reduced survival in breast, stomach and non- small cell lung cancer, thus acting as a prognostic indicator in these cancers. ${ }^{[22,23]}$ Alternatively, expression of wild type of p53 can induce cell death or growth arrest. ${ }^{[24,25]}$

Mutations occurring in TP53 gene are one of the most common and extensively analysed molecular change in cancer research. Presence of TP53 mutation in lung, colorectal, prostate and breast carcinomas is associated with poor prognosis and with chemoresistance. ${ }^{[3]}$ TP53 mutation, commonly occur in ovarian high-grade tumors. However, according to Russel Vang et al, immunohistochemistry cannot accurately predict TP53 mutation status. ${ }^{[26]}$

According to Anna Yemelyanova et al., who compared TP53 mutation and p53 immunohistochemical expression, they observed that diffuse positive staining and complete absence of staining were associated with TP53 gene mutation. Thus, they concluded that immunohistochemical staining for p53 can be used as a surrogate marker for indicating TP53 mutation in ovarian carcinoma. ${ }^{[3]}$

Present study observes that Mean p53 staining of malignant surface epithelial ovarian tumors was statistically significantly higher than that of benign tumors ( $\mathrm{p}$ value $<0.05$ ). A similar observation was seen in High grade surface epithelial ovarian carcinomas compared to Low grade tumors.

Studies that compare the survival period between low-grade and high-grade ovarian surface epithelial carcinomas are being carried out. According to J. Jonathan, O. Herod., et al., survival was poorer in patients with tumors having high expression of $\mathrm{p} 53 .{ }^{[27]}$

According to Malpica et al, in applying 2 - tier grading system, the survival of patients with low grade tumors were significantly higher than survival of patients with high grade tumors in ovarian surface epithelial carcinomas. They also observed that median survival was 1.7 years for patients with high-grade tumors compared to 4.2 years for patients with low grade tumors. ${ }^{[28]}$

According to Seidman et al, who also applied two-tier grading system, they found that 5 year survival rates for low- and high grade serous carcinomas were $56 \%$ and $34 \%$ respectively. ${ }^{[29]}$ 


\section{Conclusion}

6.1 Ovarian cancer represents the sixth most commonly diagnosed cancer among women in the world, and causes more deaths per year than any other cancer of the female reproductive system. ${ }^{[4]}$

The age-standardised incidence of ovarian cancer among 30 to 64 year old women is reasonably stable overall, with the proportion of total female cancer incidence remaining at $7 \%$ over time. ${ }^{[30]}$ Ovarian cancer is the second leading cause of cancer related death in women worldwide. ${ }^{[31]}$

Inspite of the high incidence and mortality, etiopathogenesis of this disease remains to be clearly elucidated. Recently, a 2 -tier grading system has been proposed for ovarian carcinoma subdividing it into lowgrade and high grade types. Underlying pathogenesis, clinico-pathological and immunohistochemical features are different in low grade ovarian carcinoma as compared to high grade ovarian carcinoma.

6.2 Angiogenesis is a vital step for tumor growth and metastasis. Angiogenesis can be estimated by assessing the Mean microvessel density and Mean CD34 intensity index. Studies relating to CD34 expression in low grade surface epithelial ovarian carcinoma compared to high grade ovarian surface epithelial carcinomasand in benign surface epithelial tumors of ovary are limited. Hence, there is lack of knowledge, regarding this aspect of angiogenesis in ovarian surface epithelial tumors.

Present study observes that Mean microvessel density and mean CD34 intensity index were statistically significantly higher in high grade ovarian surface epithelial carcinoma as compared to low grade ovarian surface epithelial carcinomas. This implies that high grade surface epithelial ovarian carcinomas are associated with higher vascular proliferation than low grade surface epithelial ovarian carcinomas. Similarly, CD34 and p53 expression was lower in benign surface epithelial tumor of ovary compared to malignant surface epithelial tumors.

This observation attributes to the fact that biological behaviour of high grade ovarian surface epithelial carcinomas, in terms of local invasion, nodal and distant spread can be predicted. Thus novel therapeutic targets against vascular proliferation should be identified to improve patient survival. Also, routine use of CD34 expression as a prognostic marker should be analysed and supported with further studies involving larger sample size.

6.3 Abnormalities of TP53 are the most common genetic abnormality found in malignant tumors. ${ }^{[32]}$ The product of TP53 tumor suppressor gene is p53 which is a nuclear protein that is involved in the control of cell cycle, apoptosis and maintainance of genomic stability. This p53 protein can be identified by immunohistochemical technique. ${ }^{[33]}$ p53 staining can be used as a surrogate marker for mutational status of p53 gene in ovarian carcinomas, with tumors that are diffusely positive (with $60 \%$ of cells) and those that are completely negative being indicative of TP53 mutation. ${ }^{[3]}$ However, studies comparing p53 expression in low grade malignant surface epithelial ovarian tumors versus high grade malignant surface epithelial ovarian tumors are limited.

In present study p53 expression was statistically significantly higher in high grade ovarian carcinoma as compared to low grade ovarian carcinoma. This correlates with the postulated pathogenesis of High grade ovarian tumors that arise from inclusion cysts, tubal epithelium which progresses to serous tubal intraepithelial carcinoma and then to invasive carcinoma in a faster pace compared to Low grade ovarian carcinoma. Low grade ovarian carcinoma arises from cystadenoma (or) endometriotic foci which later progresses to borderline tumors and then to invasive carcinoma at a slower pace, thus correlating with our result of lower positivity of p53. Observations made in the present study correlates well with the proposed pathogenesis of Low grade and High grade Ovarian tumors.

Moreover, CD34 and p53 expression is statistically significantly higher in malignant surface epithelial ovarian tumor compared to benign surface epithelial ovarian tumor.

Also, identification of p53 protein by immunohistochemical technique can aid in categorising malignant surface epithelial ovarian tumors into low grade and high grade thus facilitating different treatment protocol for such patients. Hence, p53 analysis could be used as a diagnostic tool in categorising ovarian surface epithelial carcinomas into low grade and high grade. However, further studies with follow up could be performed to analyse the utility of p53 expression as a prognostic marker in routine patient treatment protocol formulation. Thus, present study concludes that CD34 and p53 expression is increased in High grade malignant surface epithelial carcinoma of ovary when compared to Low grade malignant surface epithelial carcinoma of ovary.

\section{Acknowledgements}

This research was supported in part by Tamilnadu State Research Committee, King Institute of Preventive Medicine \& Research, Guindy, Chennai- 600 032. The Department of Pathology appreciates the administrative support of Dean Dr.A.Edwin Joe M.D.,B.L., Coimbatore Medical College, Tamilnadu, India towards conducting this research project. 


\section{Conflict of interest:}

The authors declare that there is no conflict of interest.

\section{Images:}

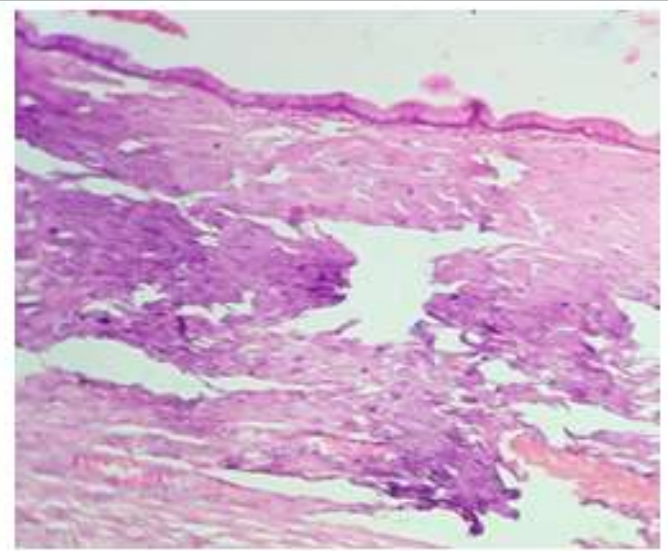

Fig 1: Benign Mucinous Cysta denoma $\mathrm{H} \& \mathrm{E}$ staining $(\mathrm{x} 10 \mathrm{X})$

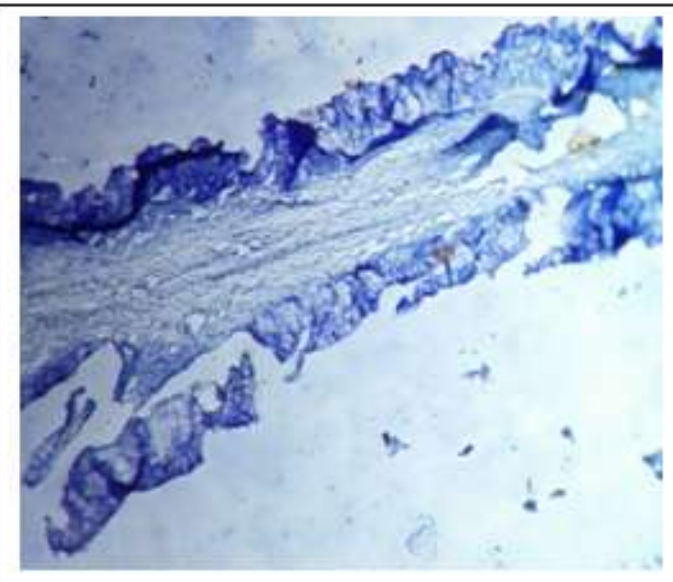

Fig 3: Benign Mucinous cystadenoma p53 staining negative Grade 1 ( $x$ 10X)

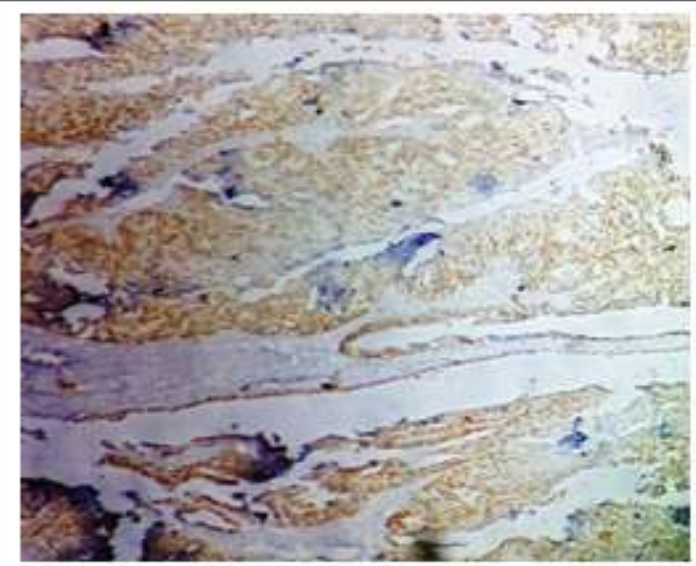

Fig 5: Mucinous cystadenocarcinoma low grade p53 staining $100 \%$ positive Grade 4 (x 10X)

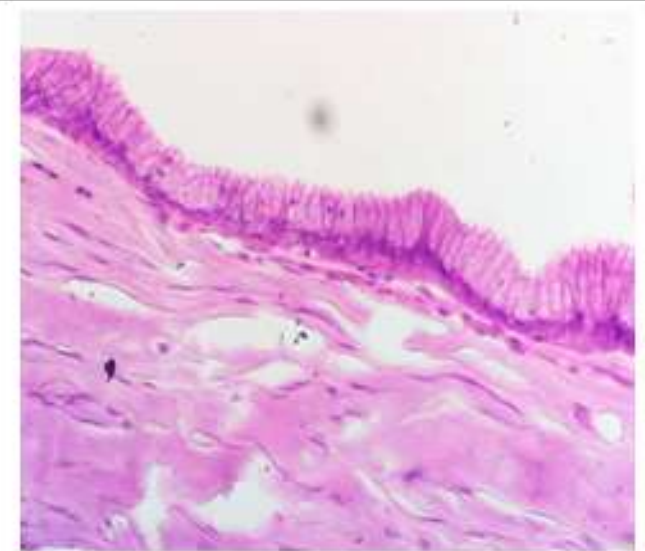

Fig 2: Benign Mucinous Cysta denoma $\mathrm{H} \& \mathrm{E}$ staining $(\mathrm{x} 40 \mathrm{X})$

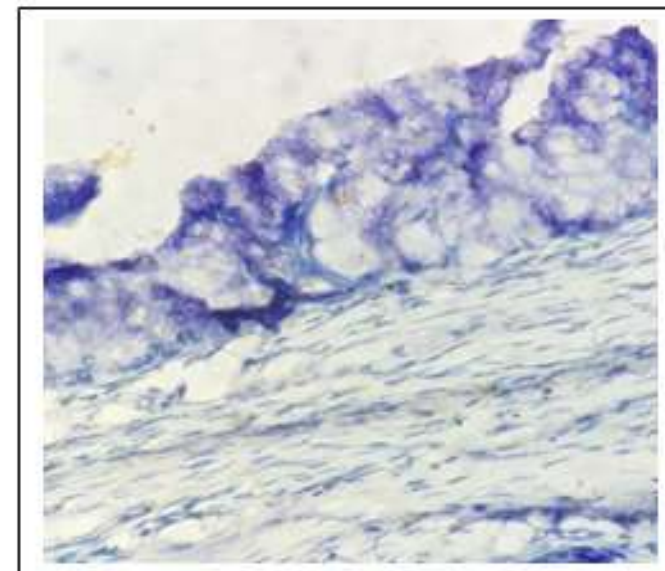

Fig 4: Benign Mucinous cysta denoma p53 staining Negative Grade 1 (x 40 X)

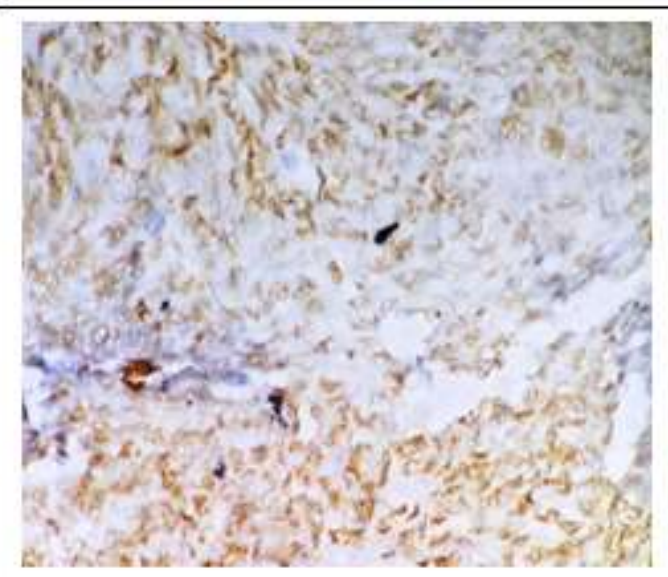

Fig 6: Mucinous cysta denocarcinoma Low Grade p 53 staining $100 \%$ positive Grade 4 (x40X) 


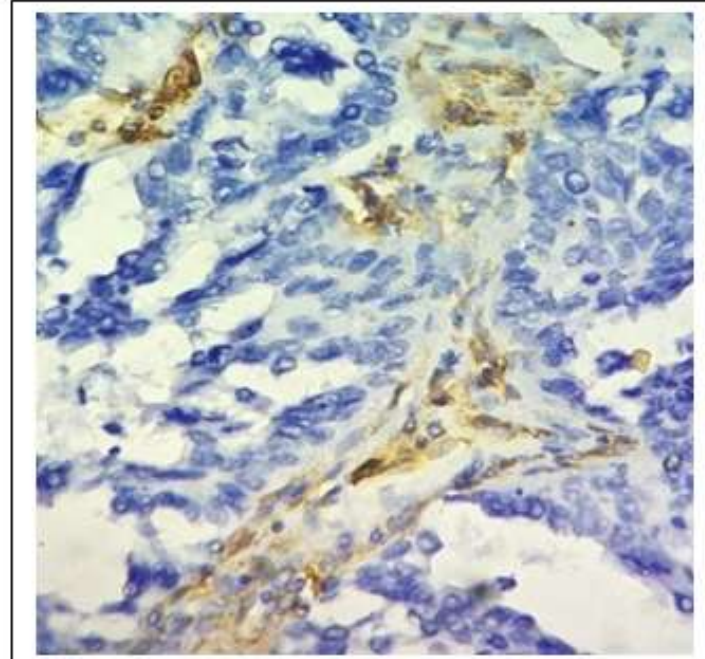

Fig 7: Mucinous cystadenocarcinoma Low grade CD34 Positive( $\mathrm{x} 40 \mathrm{X}$ )

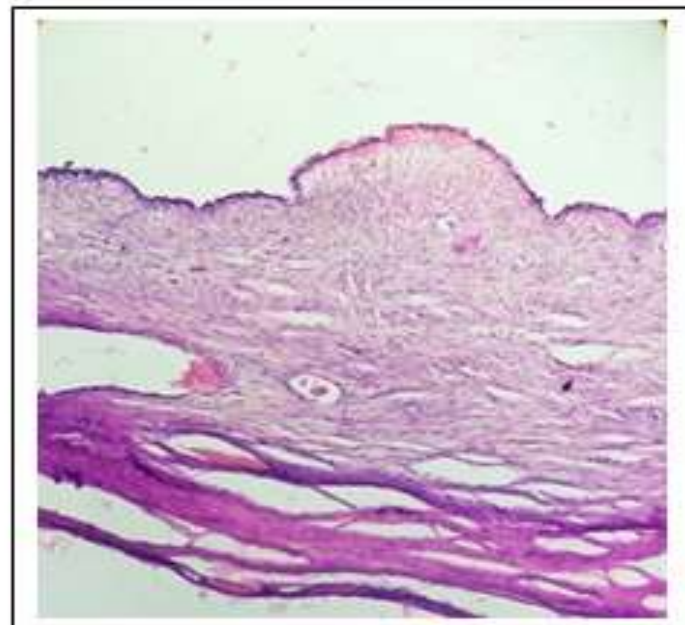

Fig 9 : Serous cystadenoma $\mathrm{H} \& \mathrm{E}(\mathrm{x} 10 \mathrm{X})$

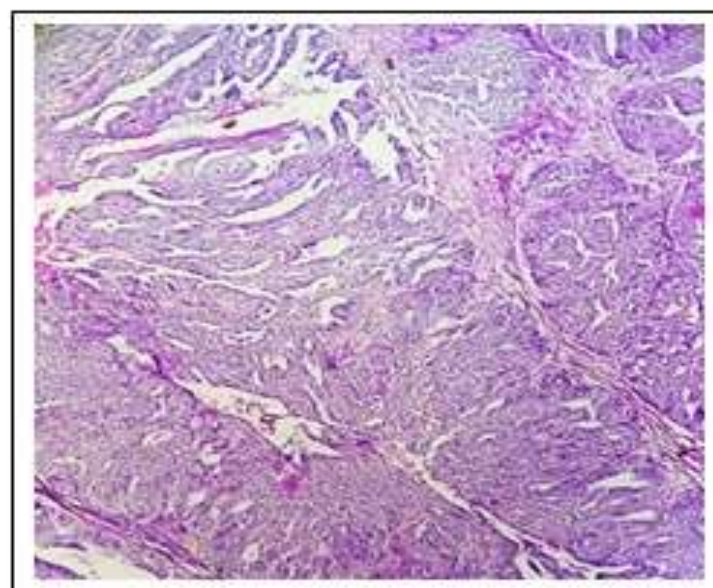

Fig 11 : Serous cystadenocarcinoma High GradeH \& E ( $x$ 10 X)

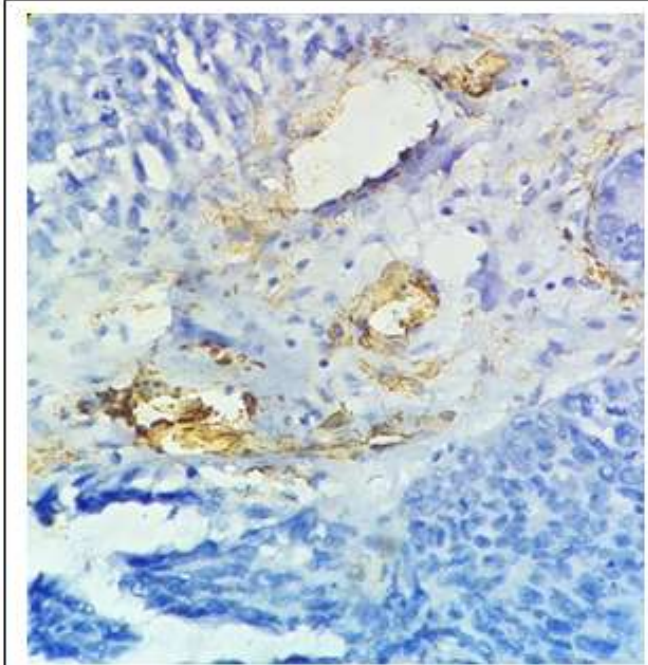

Fig 8: Mucinous cystadenocarcinoma Low Grade CD34 Positive (x 10X)

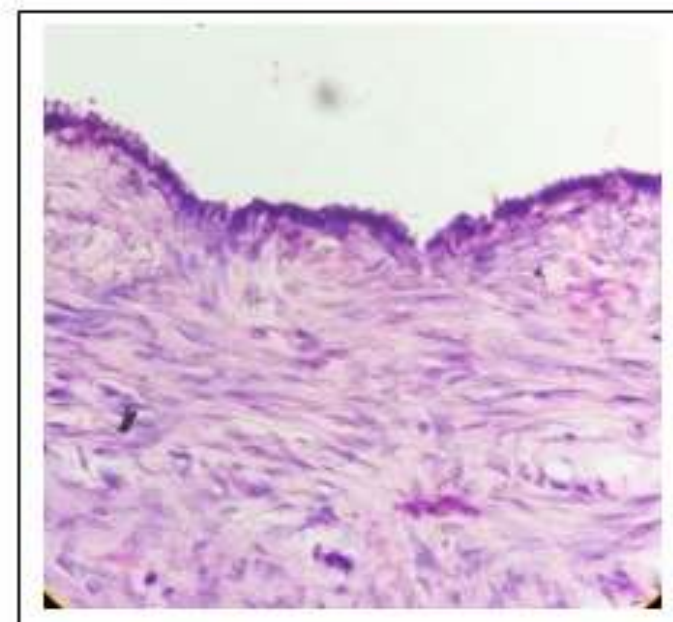

Fig 10 : Serous cystadenoma $\mathrm{H} \& \mathrm{E}(\mathrm{x} 40 \mathrm{X})$

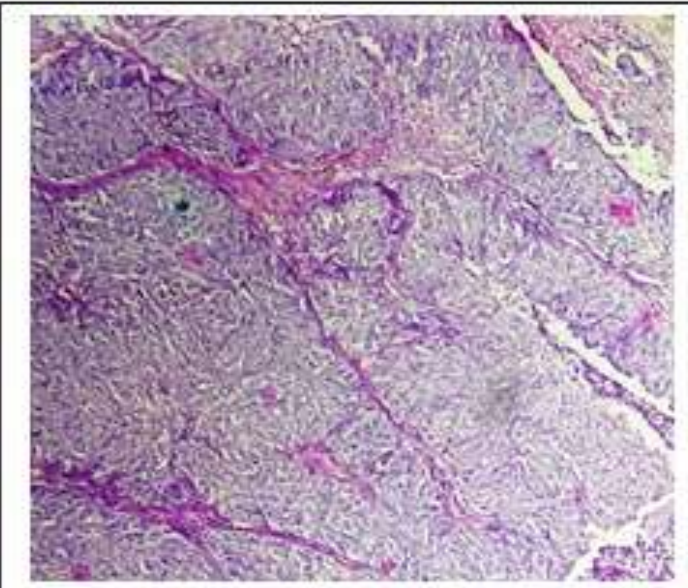

Fig 12: Serous Cystadenocarcinoma High GradeH \&E ( x 10 X) 

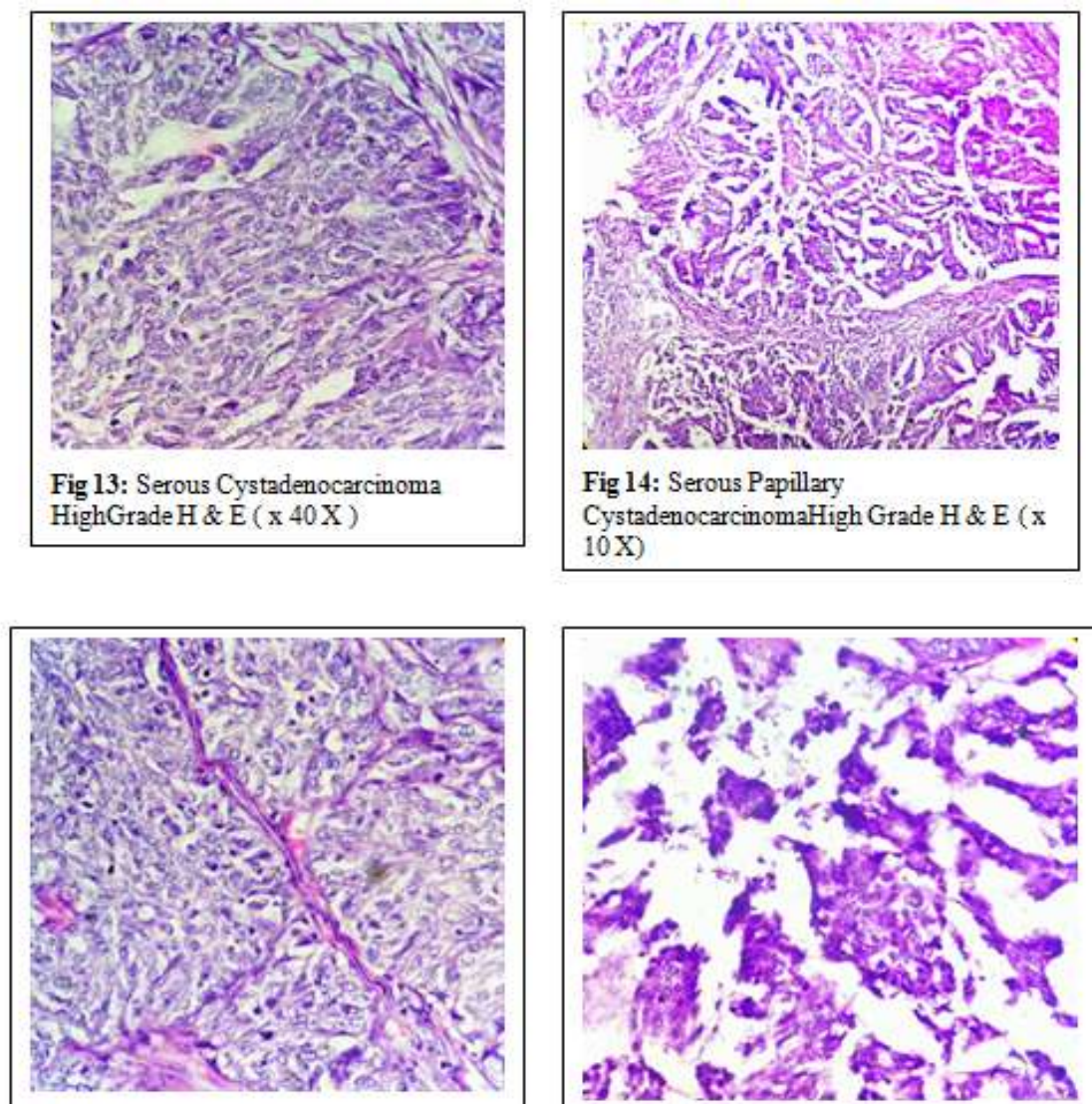

Fig 15: Serous Papillary

CystadenocarcinomaHigh Grade $\mathrm{H} \& \mathrm{E}$ ( $\mathrm{x}$ $40 \mathrm{X})$

Fig 16: Serous Papillary

CystadenocarcinomaHigh Grade $\mathrm{H} \& \mathrm{E}$ ( $\mathrm{x} 10$ $\mathrm{X})$

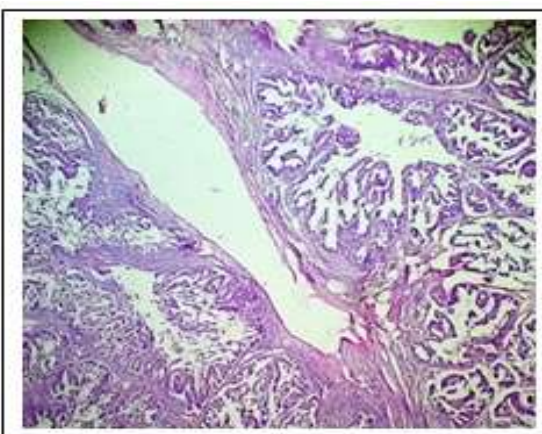

Fig 17: Serous Papillary

Cystadenocarcinomawith stromal invasion High Grade $\mathrm{H} \& \mathrm{E}$ ( $\mathrm{x} 10 \mathrm{X}$ )
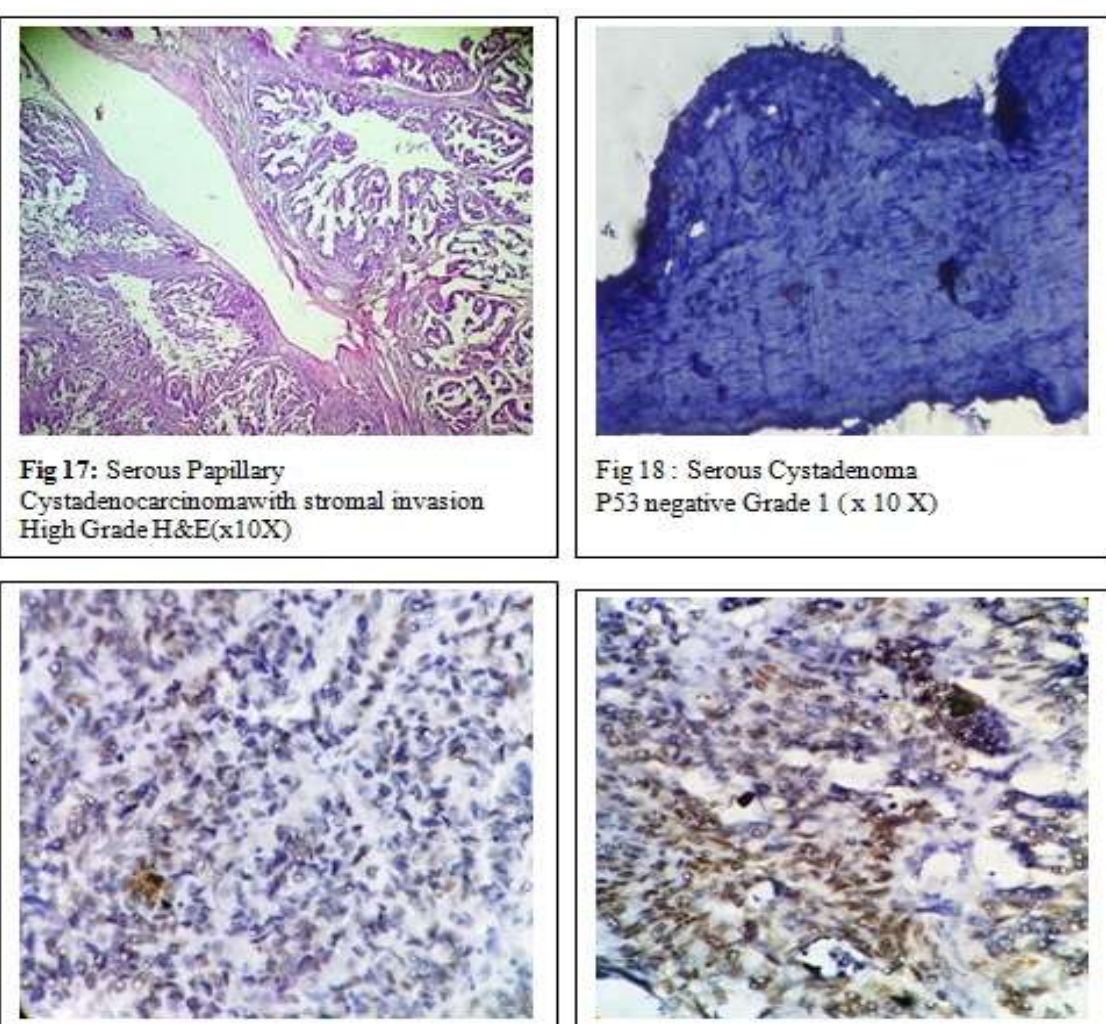

Fig 19 : Serous Cystadenocarcinoma Low Grade P53 $30 \%$ positive Grade 2 ( $\mathrm{x} 40 \mathrm{X}$ )

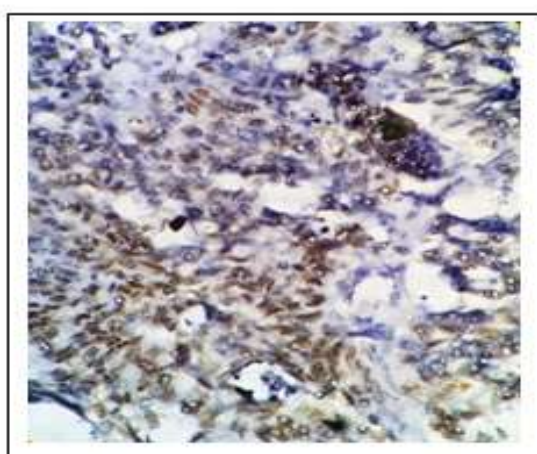

Fig 20 : Serous Cystadenocarcinoma High Grade $53370 \%$ positive Grade 4 (x $40 \mathrm{X}$ 


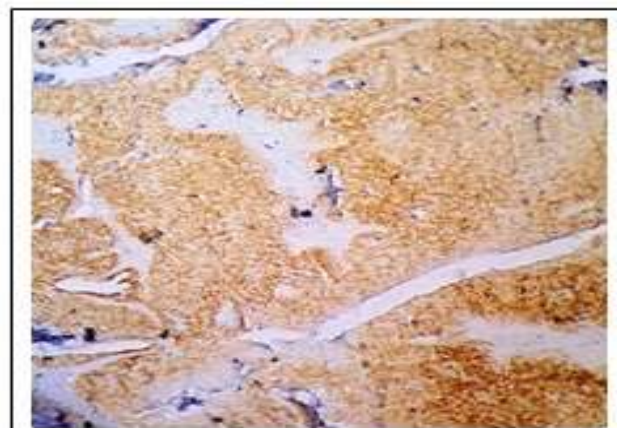

Fig 21 : Serous Cystadenocarcinoma High Grade $\mathrm{p} 53100 \%$ positive Grade 4 ( $\mathrm{x} 10 \mathrm{X}$ )

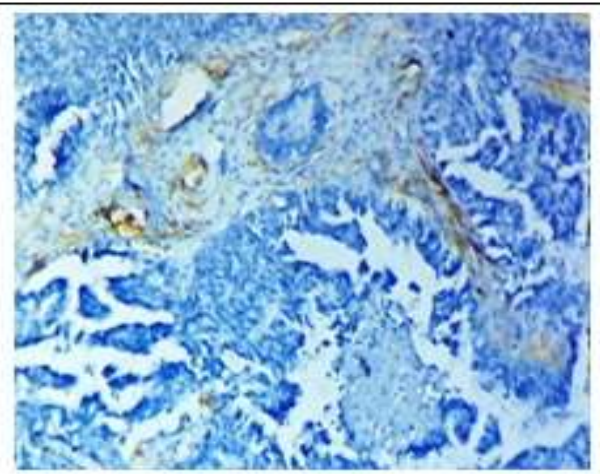

Fig 23: Serous Papillary Cystadenocarcinoma High Grade CD 34 positive ( $10 \mathrm{X}$ )

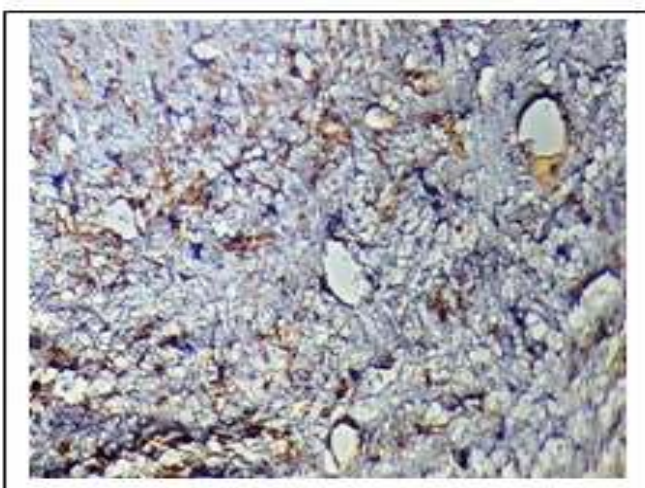

Fig25: Serous Cystadenocarcinoma HighGrade CD 34 positive $(x, 10 \mathrm{X})$

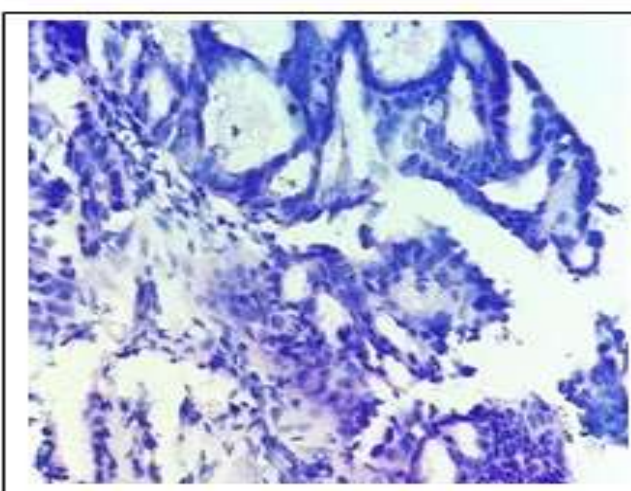

Fig 27: Endometrioid carcinoma Low Grade $\mathrm{H}$ \& E stain ( $\mathrm{x} 40 \mathrm{X}$ )

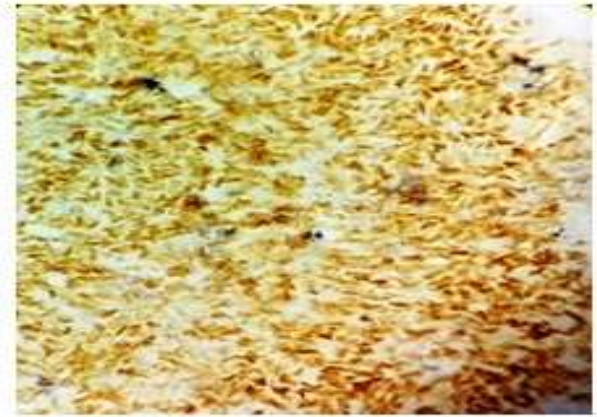

Fig 22 : Serous Cystadenocarcinoma

HighGrade $53100 \%$ positive Grade 4 (x 40 $\mathrm{X})$

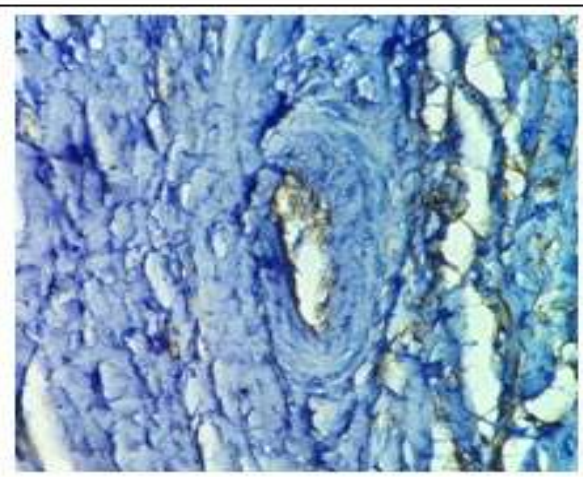

Fig 24: Serous Cystadenoma CD 34 positive ( $\mathrm{x} 40 \mathrm{X}$ )
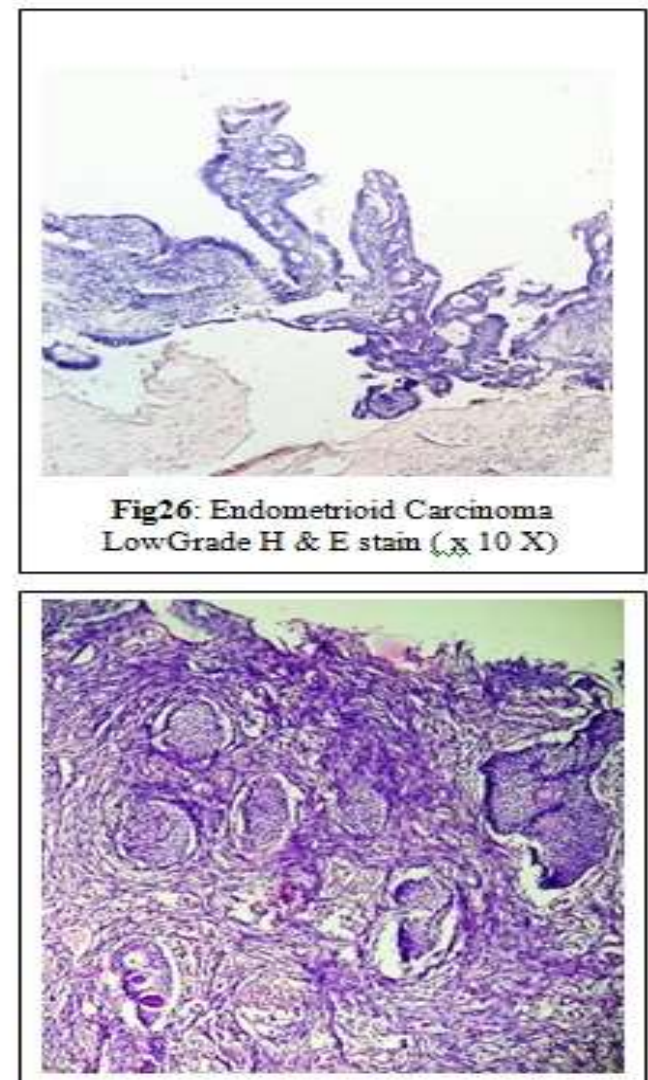

Fig 28: Brenner tumor

$\mathrm{H} \& \mathrm{E} \operatorname{stain}(\mathrm{x} 10 \mathrm{X})$ 

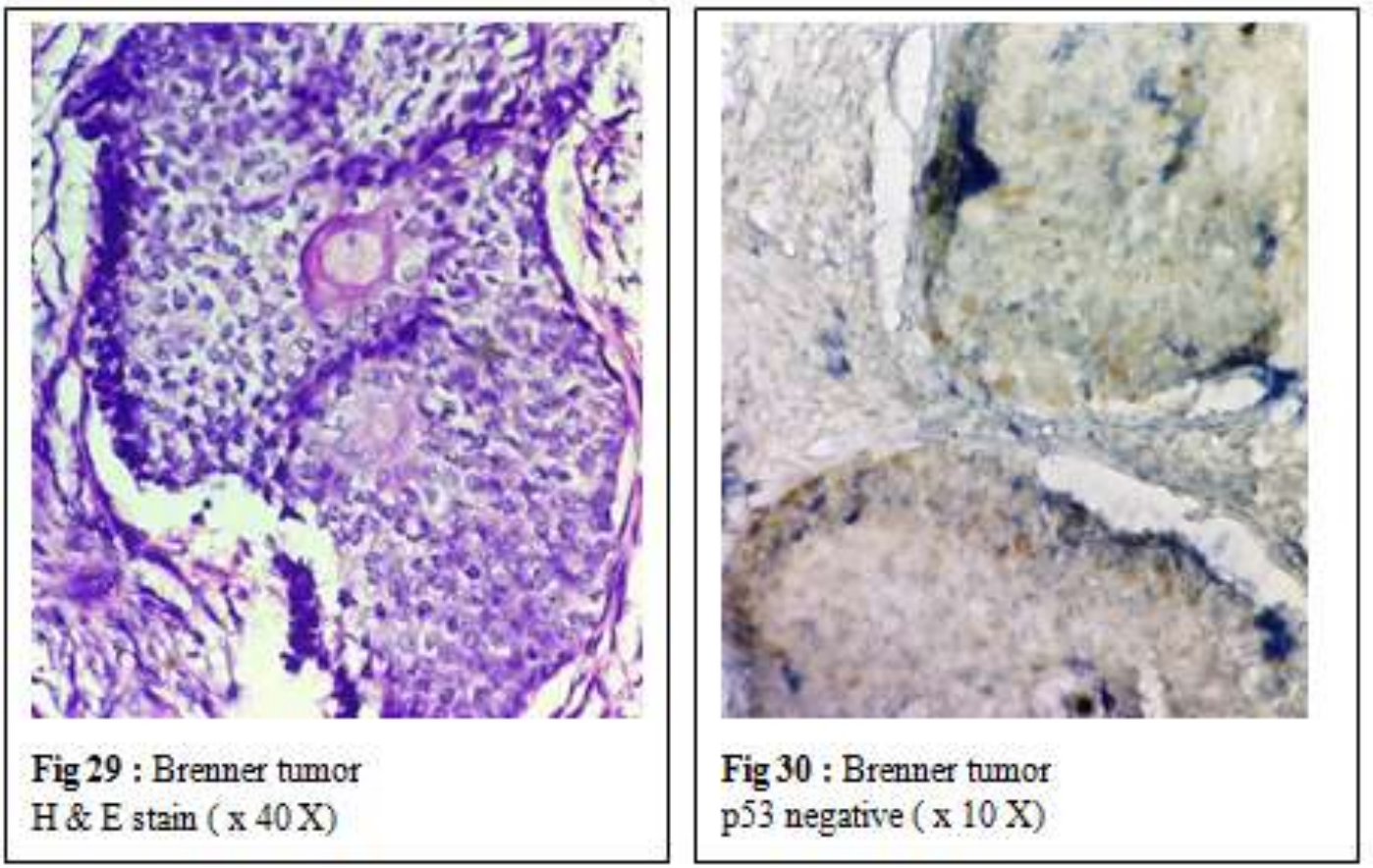

\section{References}

[1]. Kumar, Abbas, Aster. The Female Genital Tract: Robbins and Cotran Pathologic Basis of Disease, South Asia Edition pg.1023, pg. 1029

[2]. Juan Rosai MD, Female Reproductive System, Rosai and Ackerman's Surgical Pathology, Tenth Edition, pg.1583.

[3]. Anna Yemelyanova, Russell Vang et al., Immunohistochemical staining patterns of p53 can serve as a surrogate marker for TP53 mutations in Ovarian carcinoma - An Immunohistochemical and Nucleotide Sequencing Analysis. Mod.Pathol. 2011;24(9):12481253

[4]. Jennifer Permuthwey, Thomas A. Sellers et al., Epidemiology of Ovarian Cancer, Cancer Epidemiology Vol. 472 of the series Methods in Molecular Biology pp. 413 - 497.

[5]. Tanuja Rastogi, Susan Devesa et al., Cancer incidence rates among South Asians in four geographic regions : India, Singapore, UK and US : International Journal of Epidemiology 2008; 37: 147 - 160.

[6]. Parkin DM, Whelan S, Ferlay J, Teppo L, Thomas DB. Cancer Incidence in Five continents Volume III. IARC Scientific Publication No.155.Lyon, France : International Agency for Research on Cancer ( IARC) press, 2002.

[7]. Shelly Sehaal, Prashant Goyal et al., Differences in the angiogenesis of benign and malignant ovarian surface epithelial tumors demonstrated by microvessel density and immunohistochemistry. J Interdiscipl Histopathol 2013; 1(3):145 - 152.

[8]. Christopher D.M.Fletcher, Charles J.Zaloudek, Karuna Garg, Tumors of the Female Genital Tract. Diagnostic Histopathology of Tumors, Fourth edition.

[9]. Cho K R, Shih IM (2009) Ovarian Cancer. Annu Rev Pathol Mech Dis 4:287 - 313 .

[10]. R.J.Kurman, L.Hedrick Ellenson, B.M.Ronnett (eds) Blaustein's Pathology of the Female Genital Tract (6 $6^{\text {th }}$ edition), DOI 10.1.007/978-1-4419-0489-8_14, pg 692.

[11]. Jeffrey D, Seidman, Kathleen, R.Cho, Brigitte M, Ronnett, Robert J.Kurman : Surface Epithelial Tumors of the Ovary. Blaustein's Pathology of the Female Genital Tract $\left(6^{\text {th }}\right.$ edition)

[12]. Silverberg SG. Histologic grading of ovarian carcinoma : a review and proposal. Int. J. Gynaecol Pathol. 2000;19:7 - 15

[13]. Fan J - PD , Jagger R. Bicknell R. Controlling the vasculature : angiogenesis, antiangiogenesis and vascular targeting ongene therapy. Trends Pharmacol Sci 1995;16: 57 - 66.

[14]. Klagsburm M, D Amore P. Regulators of angiogenesis. Annu Rev Physiol 1991;53:217 -2391.

[15]. Obermair A, Wasicky R, Kaider A et al. Prognostic significance of tumor angiogenesis in epithelial ovarian cancer. Cancer Lett.1999;138:175 - 82.

[16]. Heimburg S, Oehler MK, Papadoopulos T et al., Prognostic relevance of the endothelial marker CD34 in ovarian cancer. Anticancer Res 1999;19:2527.

[17]. Rossachacka - Rostalska B, Gisterek IJ, Suder E et al. Prognostic significance of microvessel density in ovarian cancer. WiadLek.2007;60(3-4);129-37

[18]. Muthuramalingam SR, Braybrooke JP, Blann AD, et al: A prospective randomised phase II trial of thalidomide with carboplatin alone as a first- line therapy in women with ovarian cancer, with evaluation of potential surrogate markers of angiogenesis. Eur $\mathbf{J}$ Gynaecol Oncol 32: 253 -258, 2011.

[19]. Matulonis UA : Bavazicumab and its use in epithelial ovarian cancer. Future Oncol 7: $365-379,2011$

[20]. Hata K, Watanabe Y, Nakai H, Hata T and Hoshiai H : Expression of the vascular endothelial growth factor (VEGF) gene in epithelial ovarian cancer : an approach to anti- VEGF therapy. Anticancer Res 31: 731-737,2011.

[21]. Lotem. J., and Sachs. L. Regulation by bcl-2, c-myc and p53 of susceptibility to induction of apoptosis by heat shock and cancer chemotherapy compounds in differentiation - competent and defective myeloid leukemic cells . Cells Growth \& Differ. $4: 41-47$, 1993

[22]. Bartek J., Bartkova J., and Vojtesek B., Aberrant expression of the p53 oncoprotein is a common feature of a wide spectrumof malignancies. Oncogene. 6:1699 - 1703. 1991

[23]. Harris A.L. Mutant p53 - the commonest genetic abnormality in human cancer ? - J. Pathol. 162: 5-6, 1990. 
[24]. Diller. L, Kassel. J., Nelson. C.E., Gryka. M.A., Litwak. G., Gebhardt. M., Bressac.B., Ozturk.M., Baker S.J. Vogelstein. B and Friend.S.H., p53 functions as a cell cycle control protein in osteosarcomas. Mol. Cell. Biol $10: 5772-5781,1990$.

[25]. Yonish - Rouach. E., Resnitzky. D.,Lotem J., Sachs.1., Kimchi. A and Oren M. Wild type p53 induces apoptosis of myeloid leukemic cells that is inhibited by interleukin 6. Nature ( Lond.),352: $345-347,1991$.

[26]. Russell Vang, Ie Ming, Robert J. kurman. Ovarian low-grade and high grade serous carcinoma : Pathogenesis, clinicopathologic and Molecular Biologic Features and Diagnostic problems. Adv. Anat. Pathol. 2009 Sep:16 (5); $267-282$.

[27]. J. Jonathan O.Herod, Aristides G.Eliopoulos, Jane Warwick, Gerald Niedobitek, Lawrence S.Young and David J.Kerr. The prognostic significance of bcl-2 and p53 expression in ovarian carcinoma. Cancer research 56.2178-2184, May 1, 1996.

[28]. Malpica A, Deavers MT, Lu K. et al., Grading ovarian serous carcinoma using a two - tier system. Am.J.Surg. Pathol. 2004;28:496 -504 .

[29]. Seidman JD, Horkayne - Szakaly I, Cosin JA et al. Testing of two binary grading systems for FIGO stage II serous carcinoma of the ovary and peritoneum. Gynaecol Oncol. 2006;103;70

[30]. P.K.Dhillon, BB Yenle, R.Dikshil, A.P.Kurkure and F.Bray. Trends in breast, ovarian and cervical cancer incidence in Mumbai, India over a 30 year period, 1976 -2005: an age-period - cohort analysis.Br J Cancer 2011 Aug 23,105(5) 723 -730.Published Online 2011 Aug 9; doi : 10.1036 bjc 2011.301

[31]. Laurartiu Pirtea, Marius Paica, Anca Maria Cimpean. Endothelial Cell Activation \& Proliferation in Ovarian tumours: Two distinct steps as potential markers for anti-angiogenic therapy response. Molecular Medicine Reports 5:1181-1184,2012.

[32]. Chang F, Syrjanen S, Syrjanen K. Implications of the p53 tumorsuppressor gene in clinical oncology. J Clin Oncol 1995,13:10091022 .

[33]. Juan Rosai MD : Special techniques in surgical pathology: Rosai and Ackerman's Surgical Pathology. Tenth Edition.pg 58,67 